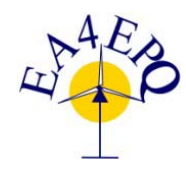

International Conference on Renewable Energies and Power Quality (ICREPQ'14) Cordoba (Spain), $8^{\text {th }}$ to $10^{\text {th }}$ April, 2014

Renewable Energy and Pourer Qualiuy. Fournal (RE\&PQJ)

ISSN 2172-038 X, No.12, April 2014

\title{
Estimation and Forecast of Wind Power Generation by FTDNN and NARX-net based models for Energy Management Purpose in Smart Grids
}

\author{
A. Di Piazza, M. C. Di Piazza and G. Vitale \\ Consiglio Nazionale delle Ricerche (CNR) \\ Istituto di Studi sui Sistemi Intelligenti per l'Automazione (ISSIA) UOS Palermo \\ Via Dante, 1290141 Palermo (Italy) \\ Phone: +39 091 6113513, fax: +390916113028, \\ e-mail: a.dipiazza@pa.issia.cnr.it; dipiazza@pa.issia.cnr.it, vitale@pa.issia.cnr.it
}

\begin{abstract}
This paper is focused on the prediction and forecast of climate time series, particularly useful for planning and management of the power grid, by artificial neural networks. An appropriate prediction and forecast of climate variables, indeed, improves the overall efficiency and performance of renewable power plants connected to the power grid. On such a basis, the application of suitable Artificial Neural Networks (ANNs) to the field of wind power generation is proposed. In particular, two dynamic recurrent ANNs, i.e., the Focused Time-Delay Neural Network (FTDNN) and the Nonlinear autoregressive network with exogenous inputs (NARX), are used to develop a model for the estimate and forecast of daily wind speed. Results, applied to a turbine model, allow the produced power to be calculated for energy management and planning purpose in smart grids.
\end{abstract}

\section{Key words}

Forecast, Neural Networks, Wind generation, Energy management, Smart grids.

\section{Introduction}

The evaluation of the renewable energy potential production capability is of paramount importance to achieve the optimal exploitation of the available resources. This is especially beneficial in a smart grid context where an optimal energy management can be achieved by a complete dataset and data forecast. However, datasets can be corrupted for missing data or errors in some time intervals, whereas forecast is based on long term data sampling. For these reasons, a tool for data rebuilding (prediction) or data forecast is advantageous for energy and economical planning. In particular, the variable considered in this work is the wind speed whose accurate prediction is helpful for the reliable and high-quality operation of power system, and reduces the operating costs of wind power generation [1].

The wind speed prediction can also let energy stakeholders predict the output power of a generator in advance, and can effectively reduce the impact of wind power fluctuations on the power grid. This allows the overall efficiency and power quality of the plant to be increased, which is a pivotal issue when designing and operating any renewable-based power generator [2]-[3].

In addition, the energy forecast is useful to give the input to intelligent EMS (Energy Management System) to optimally manage loads and storage systems [4]-[6].

With reference to wind-farm power generation, modelling wind speed by means of time series forecasting techniques is going to be considered more and more promising [1], [7]. The high complexity and the intermittent and non-linear behaviour of the wind speed makes necessary the use of autoregressive, fuzzy and neural techniques and forecast model based on hybrid algorithms [8]-[10].

In general, Artificial Neural Networks (ANNs) and hybrid algorithms provide to be more effective than other classical autoregressive predictors for both wind speed and solar radiation [1], [7],[11],[12]. On such a basis, this paper proposes the use two dynamic neural networks, i.e., the Focused Time-Delay Neural Network (FTDNN) and the nonlinear autoregressive network with exogenous inputs (NARX Network), with their training algorithms, to obtain the estimation and forecast of daily wind speed. The same approach has been previously used by the authors for estimation and forecast of the daily solar radiation, with very good results [13]-[14].

The proposed models are experimentally validated and a comparison between them is given as well.

\section{Purpose of the work}

This paper proposes an application of ANNs to the field of wind power generation considering its high rate of penetration in the context of smart grids and distributed generation. In particular, two suitably trained dynamic recurrent ANNs, described in the next section, are used to develop a method for prediction and forecast of daily wind speed. The ANNs implemented in this study are particularly suitable to the time series prediction and show better performance respect to the classical feedforward ANNs in several similar applications [15]-[16]. 
The higher computational demand, anyway affordable by a common PC, is justified by the possibility to obtain more reliable and precise models.

The geographical area under consideration is situated in Sicily, the biggest island of Mediterranean area. In particular, the used dataset represents the daily wind speed and the daily maximum and minimum temperature recorded during 3 years, from 2010 to 2012.

All data were provided by SIAS (Servizio Informativo Agrometeorologico Siciliano).

The normalized Root Mean Square Error (nRMSE) and the Coefficient of Variation of the Root Mean Squared Error, $\mathrm{CV}(\mathrm{RMSE})$, are used to define the deviation between observed and estimated values. They are defined, respectively as:

$$
\begin{aligned}
& n R M S E=\frac{1}{Y_{\max }-Y_{\min }} \sqrt{\frac{\sum_{i=1}^{N}\left(Y_{i}-\hat{Y}_{i}\right)^{2}}{N}} \\
& C V(R M S E)=\frac{1}{\bar{Y}} \sqrt{\frac{\sum_{i=1}^{N}\left(Y_{i}-\hat{Y}_{i}\right)^{2}}{N}}
\end{aligned}
$$

where $Y$ is the original time series, $\hat{Y}$ is the predicted time series, $Y_{\max , \min }$ are the maximum and minimum observed values and $\bar{Y}$ is the mean of the observed values.

In this paper, the tables that shows the performance indices will contain the name of the indices with subscripts $t, r$ and $f$ that indicate respectively training phase (application of models to estimation set), recall phase (application of models to validation set) and forecast phase.

\section{Modelling Approach}

The chosen ANNs are the Focused Time-Delay Neural Network (FTDNN) and the nonlinear autoregressive network with exogenous inputs (NARX Network). These ANNs can be both classified as recurrent dynamic ANNs. The specific feature of such neural networks respect to static feed-forward networks, such as backpropagation (BP) ANN or cascade-forward ANN, is their capability to learn dynamic or time series relationships. In particular, in dynamic ANNs, the output depends not only on the current input but on the current and previous inputs, outputs or states of the network, as well.

With specific reference to the NARX network, a preliminary analysis, based on the system identification methodology has been carried out. It allows the best mathematical representation of the dynamic relationship among the studied variables (temperature and wind speed) by linear models to be identified. In addition, this analysis allows the limitations of linear models to be highlighted and gives a justification for using the NARX. A description of the used ANNs, including the system identification-based analysis, is given hereinafter [15].

\section{A. Focused Time-Delay Neural Network (FTDNN)}

The FTDNN is the most straightforward dynamic network, which consists of a feed-forward network with a tapped delay line at the input. This is part of a general class of dynamic networks, called focused networks, in which the dynamics appear only at the input layer of a static multilayer feed-forward network. This network is well suited to time-series prediction. The FTDNN can be trained so to perform either one-step-ahead predictions or multistep-ahead predictions. In this last case the predictions are fed back to the input of the network, continuing to iterate.

The structure of a FTDNN is shown in Fig 1.

The network has been trained by the conjugate gradient backpropagation with Polak-Ribiére updates and the initial weights have been extracted randomly from a standardized normal distribution.

The value of the learning rate, initially equal to 0.01 , decreases according to an exponential law. The epochs used for training phase were 1000. Three network architectures, having different number of neurons in the hidden layer, have been evaluated and compared by means of statistical indices both in training and recall phases, for different values of input delays.

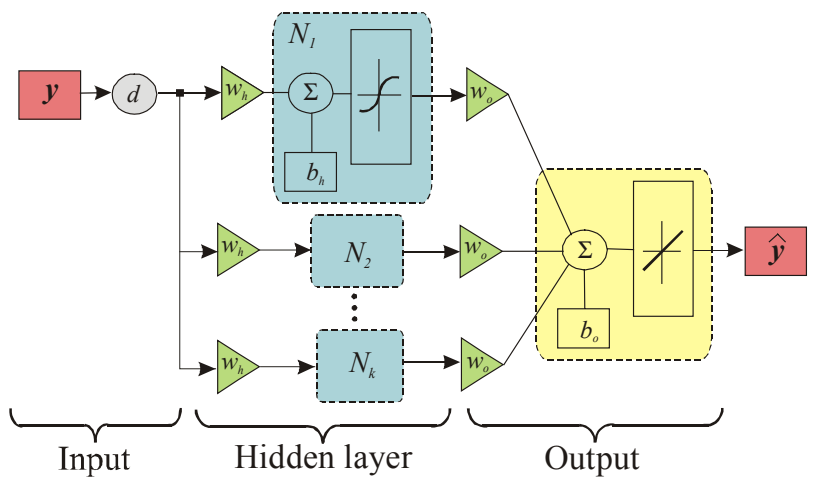

Fig.1 Structure of FTDNN.

\section{B. System identification}

System identification is a methodology for building mathematical models of dynamic systems using measurements of the system's input and output signals [16]. Dynamic system models can be defined as systems that have internal dynamics or memory of past states. In this application, daily temperature and daily wind speed data are taken into account and an analysis to find the linear relationship between them is performed. In a dynamic systems the values of the output signals depend on both the instantaneous values of its input signals and also on the past behavior of the system. In this study, the output of the process is the wind speed and the input is the temperature. In particular, a time series of daily wind speed and a time series of daily temperature in the period 2010-2012 are available. Each dataset is split into two parts: an estimation set $(80 \%)$ and a validation set $(20 \%)$. A preprocessing procedure is preliminary applied to temperature and wind speed data. In particular, the detrending is applied to datasets and the outputs are reverse-transformed back into the units of the original 
target data. Then, different linear models, among those available within the Matlab System Identification Toolbox, are selected and different model structures are tested. From the comparison of different models, the best estimation model is the ARX, an autoregressive exogenous model. It can be mathematically represented as:

$$
\begin{aligned}
& y(t)+a_{1} y(t-1)+\ldots+a_{n a} y(t-n a)= \\
& =b_{1} u(t-n k)+\ldots+b_{n b} u(t-n b-n k+1)+e(t)
\end{aligned}
$$

The parameters na and nb are the orders of the ARX model; nk is the delay (number of input samples that occur before the input affects the output); $y(t-1)+\ldots+y(t-n a)$ is previous outputs on which the current output depends; $u(t-n k)+\ldots+u(t-n b-n k+1)$ is previous and delayed inputs on which the current output depends; $e(t)$ is a white-noise disturbance.

Once the best linear model has been identified, three different structures of the ARX model, having different parameters, have been considered. In particular, the chosen structure are: the $\operatorname{arx} 441(n a=4, n b=4$ and $n k=1)$, the $\operatorname{arx} 442(\mathrm{na}=4, \mathrm{nb}=4, \mathrm{nk}=2)$ and the arxqs. This last structure is the so called fourth-order autoregressive with nk estimate by inpulse function, that calculates the unit impulse response of a dynamic system model.

Although the ARX model is the best linear representation of the dynamic system temperature/wind speed relationship, it does not give satisfactory results, as it will be explained in Section 4. For this reason, a different approach, i.e., the use of a suitable ANN (the NARXnet), is followed. With this approach the strong non linearity of the temperature/wind speed relationship and the variability of the wind speed are appropriately taken into account.

\section{Nonlinear autoregressive network with exogenous inputs (NARX)}

The NARX network, differently from the focused networks, is a recurrent dynamic network, with feedback connections enclosing several layers of the network. The NARX model is based on the linear ARX model, it is well suited to model nonlinear dynamic systems and is commonly used in time-series modeling thanks to its adaptive learning process also with small scale meteorological data, collected, for example in less than one year [17].

In this network the next value of the dependent output signal $y(t)$ is regressed on previous values of the output signal and previous values of an independent (exogenous) input signal. A feed-forward neural network, such as a standard Multilayer Perceptron (MLP), can be used to approximate the nonlinear mapping function $f$. As for the training of the NARX network, it is noteworthy to highlight that the true output is available during the training of the network. Therefore, this value can be used instead of the estimated output during the training phase.

This feature realizes a series-parallel architecture in whose main advantages are: a more accurate input to the feed- forward network and the possibility to adopt a purely feed-forward architecture, and static backpropagation [18].

For the application considered in this paper, the NARX network, where the exogenous input is the temperature, has been implemented using two configurations: a seriesparallel architecture and parallel architecture.

The former is used in two operating modes:

1. the temperature and wind speed datasets are divided into estimation sets and validation sets, to assess the network performance;

2. all data, except those belonging to the last 10 days, are used for learning the wind speed behaviour.

The latter architecture utilizes the exogenous input $x(t)$ (in this case the temperature) to forecast the wind speed. Once the first structure has been trained, the second architecture is used to perform the forecast of the daily wind speed, according to a multi-step ahead prediction. These configurations are illustrated in Fig 2. Also in this case, the used training algorithm is the conjugate gradient backpropagation with Polak-Ribiére updates. Finally, a comparison by means of statistical indices both in training and recall phases has been done to define the best model.
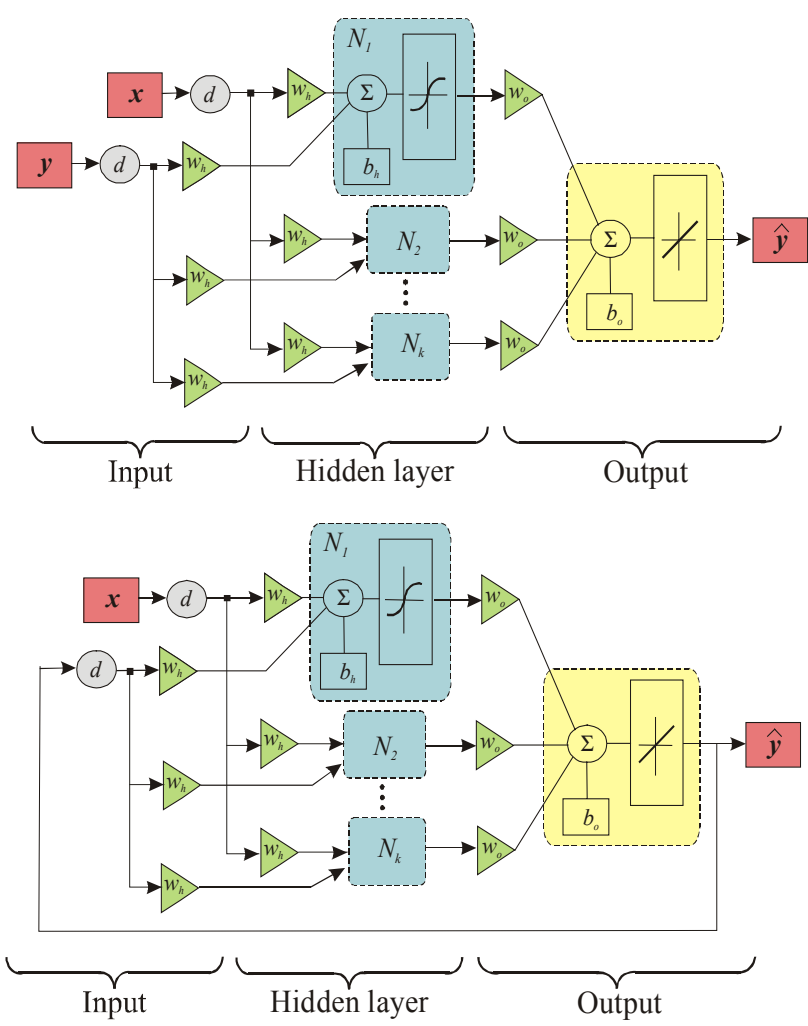

Fig. 2 Structures of the NARX network: Series-parallel, up; Parallel, down.

\section{Data measurement and preprocessing}

The daily wind speed and the daily maximum and minimum temperature registered on Palermo weather station, in the north-east of Sicily, are used as dataset to train the ANNs. These registrations give, for the considered period of time, a complete series of daily wind speeds and temperatures. As a matter of fact, to 
define models with the ability to suitably estimate and forecast measured data, the used dataset should be as good-quality as possible, homogeneous along with time and without missing data. In order to make the ANNs training more efficient, a pre-processing procedure was applied to temperature and wind speed data. In particular, a normalization step is applied to both the input vectors and the target vectors in the dataset and the network output was reverse-transformed back into the units of the original target data (post-processing procedure). In Fig. 3, the times series of daily wind speed data recorded over three year is shown. It is possible observe the high non periodic and intermittent behavior of the wind speed variable.

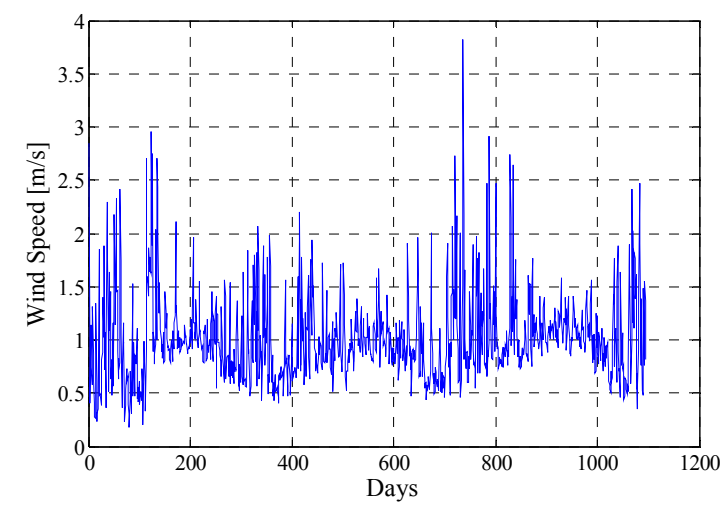

Fig. 3 Wind speed recorded at Palermo weather station over three year.

The used datasets for training the two ANNs are different. Particularly, for FTDNN the set of training and the set of validation are constructed from the measurements of two years, whereas the measurements of the third year are left for the test phase. For the System Identification application, temperature and wind speed are used and the data are split into two parts as explained in 3.B.

On the other hand, with reference to the NARX network, the set of training is formed of a time series of daily wind speed and a time series of temperature in the period 20102012. The datasets are split into estimation sets $(80 \%)$ and validation sets $(20 \%)$ for the first application with the series-parallel structure. For the second application, with series-parallel and closeloop structures, the time series of daily wind speed and a time series of temperature in the complete period are used except for the data related to the last 10 days that have been excluded from training dataset, to be used for testing the network as a forecast model.

\section{Results}

With reference to the first application, i.e. the FTDNN, three network architectures, having different number of neurons in the hidden layer, have been compared, for different values of input delays. The criteria selected to measure the performance of the neural networks are the nRMSE, and the CV(RMSE), as shown in Table 1. In particular, Table 1 gives the values of nRMSE and CV both in the training phase $\left(\mathrm{RMSE}_{\mathrm{t}}, \mathrm{CV}_{\mathrm{t}}\right)$ and in the recalling phase $\left(\mathrm{RMSE}_{\mathrm{r}}, \mathrm{CV}_{\mathrm{r}}\right)$. The best network is that where the minimum deviation between indices in the two phases is observed. As a matter of fact, the more similar are the indices the more performing is the network, by the point of view of its generalisation ability.

On the basis of such a consideration, the best structure of the neural network is the 2-5-1 with a time delay equal to 2 days. For this configuration the following indices values are obtained: $\mathrm{nRMSE}_{\mathrm{t}}=0.131, \mathrm{nRMSE}_{\mathrm{r}}=0.140$, $\mathrm{CV}_{\mathrm{t}}=0.342$ and $\mathrm{CV}_{\mathrm{r}}=0.423$. The comparison between measured (original targets) and estimated (network predicted) data is shown in Fig. 4.

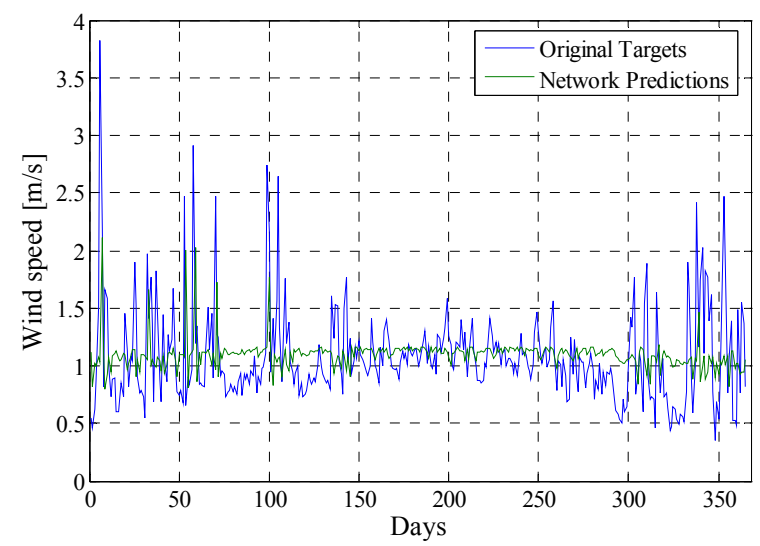

Fig. 4 Measured (original targets) and estimated (network predicted) data with FTDNN-based model.

Table 1. Assessment of the FTDNN-based model

\begin{tabular}{c|c|c|c|c|c}
\hline ANN & Delay & nRMSEt & nRMSEr & $\mathrm{CVt}$ & $\mathrm{CVr}$ \\
\hline $1-3-1$ & 2 & 0.117 & 0.145 & 0.355 & 0.378 \\
$1-3-1$ & 4 & 0.119 & 0.142 & 0.347 & 0.384 \\
$1-3-1$ & 6 & 0.274 & 0.131 & 0.317 & 0.884 \\
$1-3-1$ & 8 & 0.115 & 0.144 & 0.349 & 0.371 \\
\hline $1-5-1$ & 2 & 0.131 & 0.140 & 0.342 & 0.423 \\
$1-5-1$ & 4 & 0.124 & 0.139 & 0.338 & 0.401 \\
$1-5-1$ & 6 & 0.117 & 0.145 & 0.351 & 0.376 \\
$1-5-1$ & 8 & 0.220 & 0.118 & 0.286 & 0.710 \\
\hline $1-10-1$ & 2 & 0.148 & 0.137 & 0.335 & 0.475 \\
$1-10-1$ & 4 & 0.155 & 0.129 & 0.314 & 0.500 \\
$1-10-1$ & 6 & 0.173 & 0.118 & 0.287 & 0.556 \\
$1-10-1$ & 8 & 0.230 & 0.108 & 0.263 & 0.741 \\
\hline
\end{tabular}

As for the second application, i.e. the NARX network, it has been implemented in the two configurations (seriesparallel and parallel) described in the previous section.

For the first application the series-parallel configuration is used to evaluate the performance of NARX net in training and recall phase in comparison with ARX. It is possible to observe an improvement for nRMSE of about $14 \%$ for training phase and $12 \%$ for recall phase; and improvement of $58 \%$ for $\mathrm{CV}$ in training phase and of $31 \%$ for $\mathrm{CV}$ in recall phase. These results come from a comparison among the indices given in Tables 2 and 3 . Moreover, from a comparison of figures 5 and 6 , it is possible to note that the modelled wind speed fits much better with the experimental dataset in recall phase with the application of NARX model, respect to the use of an ARX model.

For the second application, the series-parallel configuration is used to perform the daily speed wind time series prediction and the parallel structure is used to perform the forecast of the same data. Also in this case the assessment of the method is obtained considering the nRMSE and the CV indices and the deviation between indices in phase of training and forecast. As it can be seen from Table 3, the best configuration in this case is the 2-5-1 with a time delay of 2 days with the following 
values of the statistical indices: $\mathrm{nRMSE}_{\mathrm{t}}=0.10$, $\mathrm{nRMSE}_{\mathrm{f}}=0.40, \mathrm{CV}_{\mathrm{t}}=0.143$ and $\mathrm{CV}_{\mathrm{f}}=0.435$.

Fig 7 illustrates the performance of the NARX networkbased model to predict daily wind speed and to forecast its future trend. A zoom of the forecast results is given in Fig 8 , for the sake of clarity. By the presented experimental validation it is possible to conclude that both the models provided by the two considered ANNs give good performance in predicting the average values of the wind speed.

As a result the average power generation of a given wind generator can be estimated on the basis of the following relationship, where $A$ is the area of the turbine rotor, $\rho$ is the air density and $v$ is the wind speed [19]-[21]:

$$
P_{\text {wind }}=\frac{1}{2} A \rho v^{3}
$$

Finally, it should be observed that the NARX network gives the further advantage to allow both missing data in times series of wind speed to be retrieved and future trend of the same quantity to be forecast.

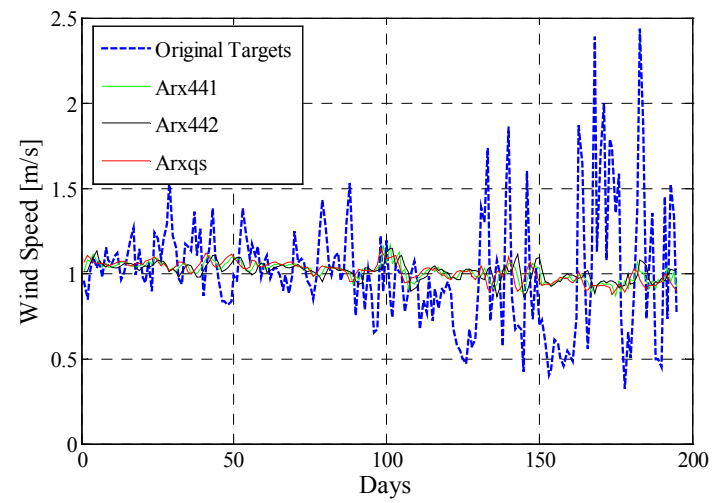

Fig. 5 Measured (original targets) and estimated (different ARX configurations) data with linear models for validation set.

Table 2 Assessment of the ARX model

\begin{tabular}{l|l|l|l|l}
\hline \multicolumn{1}{c|}{ Models } & \multicolumn{1}{c|}{ nRMSEt } & \multicolumn{1}{c|}{ nRMSEr } & \multicolumn{1}{c}{ CVt } & \multicolumn{1}{c}{ CVr } \\
\hline arx441 & 0.1177 & 0.1621 & 0.3382 & 0.4169 \\
\hline arx442 & 0.1174 & 0.1632 & 0.3405 & 0.4157 \\
\hline arxqs & 0.1184 & 0.1625 & 0.3392 & 0.4194 \\
\hline
\end{tabular}

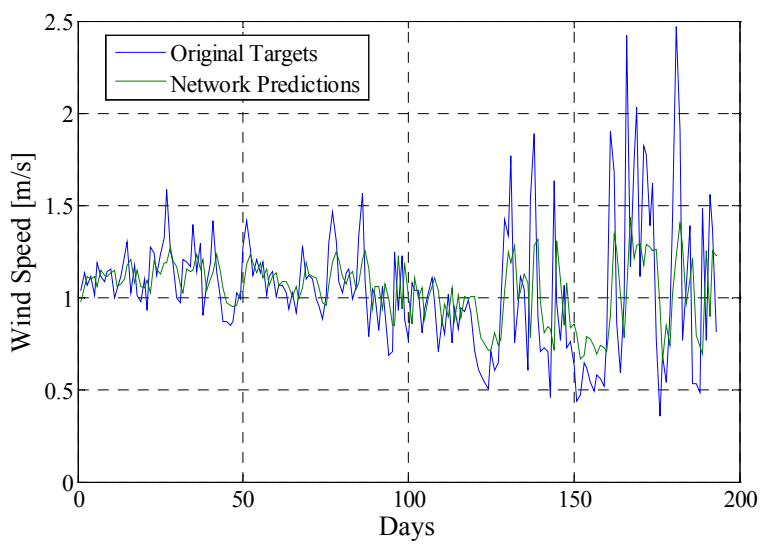

Fig. 6 Measured (original targets) and estimated (network predicted) data with NARX-based model in recall phase.
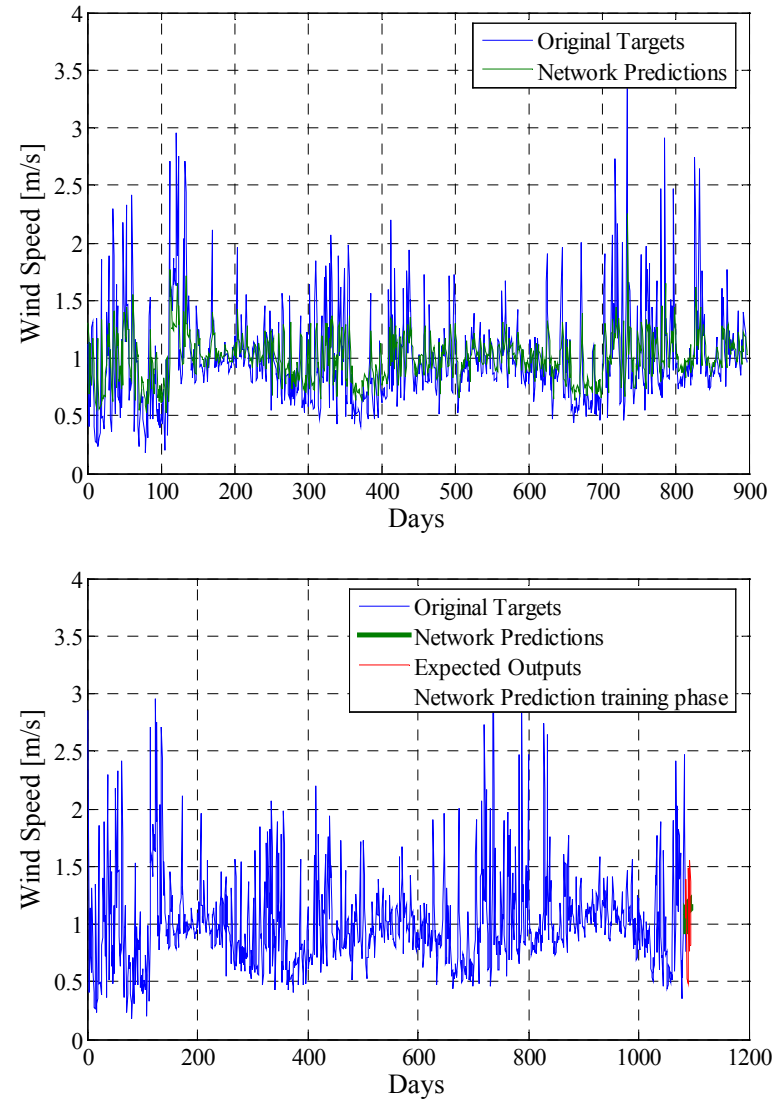

Fig. 7 Original targets and estimated data with NARXnet-based model, up; forecast with NARXnet-based model, down.

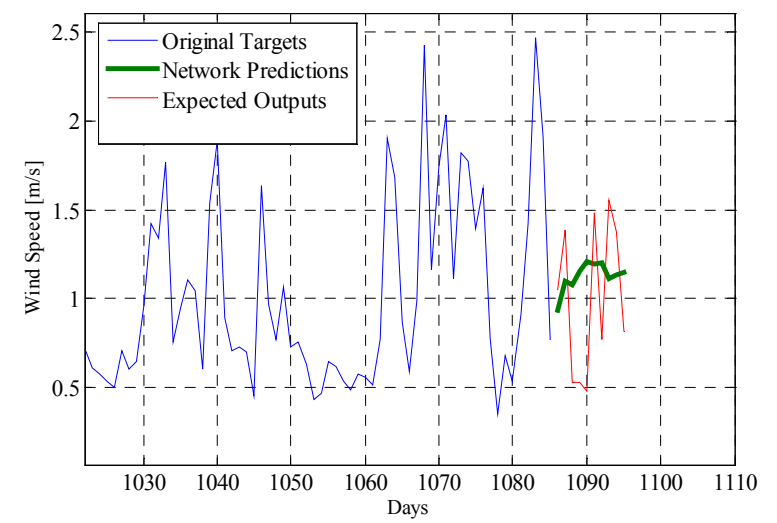

Fig. 8 Forecast with NARXnet-based model (zoom of Fig.7 down).

Table 3. Assessment of the NARXnet-based model

\begin{tabular}{c|c|c|c|c|c|c|c}
\hline ANN & Delay & nRMSEt & nRMSEr & nRMSEf & $\mathrm{CV}_{t}$ & $\mathrm{CVr}$ & $\mathrm{CVf}$ \\
\hline $2-3-1$ & 2 & 0.1002 & 0.1444 & 0.3869 & 0.1444 & 0.2912 & 0.4153 \\
$2-3-1$ & 4 & 0.1004 & 0.1444 & 0.4117 & 0.1444 & 0.2913 & 0.4420 \\
$2-3-1$ & 6 & 0.1077 & 0.1577 & 0.4453 & 0.1577 & 0.3183 & 0.4781 \\
$2-3-1$ & 8 & 0.0912 & 0.1634 & 0.4514 & 0.1634 & 0.3300 & 0.4846 \\
\hline $2-5-1$ & 2 & 0.1007 & 0.1431 & 0.4053 & 0.1431 & 0.2886 & 0.4351 \\
$2-5-1$ & 4 & 0.0943 & 0.1562 & 0.4046 & 0.1562 & 0.3151 & 0.4343 \\
$2-5-1$ & 6 & 0.0939 & 0.1425 & 0.4604 & 0.1425 & 0.2877 & 0.4943 \\
$2-5-1$ & 8 & 0.0906 & 0.1647 & 0.4376 & 0.1647 & 0.3326 & 0.4698 \\
\hline $2-10-1$ & 2 & 0.0991 & 0.1458 & 0.4417 & 0.1458 & 0.2941 & 0.4742 \\
$2-10-1$ & 4 & 0.0916 & 0.1838 & 0.5011 & 0.1838 & 0.3709 & 0.5379 \\
$2-10-1$ & 6 & 0.0814 & 0.1638 & 0.5874 & 0.1638 & 0.3307 & 0.6306 \\
$2-10-1$ & 8 & 0.0784 & 0.1894 & 0.6869 & 0.1894 & 0.3825 & 0.7374 \\
\hline
\end{tabular}

\section{Conclusions}

Two dynamic recurrent ANNs, particularly the FTDNN and the NARX network, are suitably trained and used to model wind speed both for estimation and forecast purpose. 
The daily wind speed and the daily maximum and minimum temperature in the period between 2010 and 2012 registered on Palermo weather station, in the northeast of Sicily, are used as dataset to train the ANNs The ANNs-based models are experimentally validated: they show both good performance since reliable and precise representations of daily wind speed are obtained. The followed approach gives two advantages. Firstly, it is possible to achieve a complete set of data even in case of missing data due to sensors faults or maintenance. Secondly, by exploiting forecast features, it is possible to define a suitable energy schedule together with the optimal sizing of the different parts of an electrical grid.

\section{Acknowledgement}

The authors thank SIAS (Servizio Informativo Agrometeorologico Siciliano), for delivering the wind speed and temperature measured data.

This paper has been funded by the following research projects: 1. RITmare, Italian Research for the sea CUP:B91J11000740001; 2. TESEO, High efficiency technologies for on-board energy and environmental sustainability CUP: B61C12000850005; 3. CNR per il Mezzogiorno - Advanced Technologies for Energy Efficiency and Sustainable Mobility CUP: B51J10001290001.

\section{References}

[1] Yang Xiu yuan, Xiao Yang, Chen Shu yong, “ Wind speed and generated power forecasting in wind farm", Proceedings of the CSEE. 2005; 25(11): 1-5.

[2] M. C. Di Piazza, M. Pucci, A. Ragusa, G. Vitale, "Fuzzified PI Voltage Control for Boost Converters in Multi-String PV,", IEEE 34th Annual Conference on Industrial Electronics IECON 2008, $2338-2345$

[3] G. Graditi, S. Favuzza, F. Spertino, G. Vitale, Comparison of Power Quality Impact of Different Photovoltaic Inverters: the viewpoint of the grid, IEEE International Conference on Industrial Technology, 8-10 dicembre 2004, Hammamet Tunisia

[4] M. Cirrincione, M. Cossentino, S. Gaglio, V. Hilaire, A. Koukam, M. Pucci, L. Sabatucci, G. Vitale, "Intelligent Energy Management System", 7th IEEE International Conference on Industrial Informatics INDIN 2009, 24-26th June 2009, Cardiff, UK.

[5] R. Palma-Behnke, C. Benavides,; F. Lanas, ;B. Severino, L. Reyes, J.Llanos, D. A., Saez, "Microgrid Energy Management System Based on the Rolling Horizon Strategy" IEEE Transactions on Smart Grid, Volume: 4, 2013 , Page(s): 9961006.

[6] E. Goutard, "Renewable energy resources in energy management systems", Innovative Smart Grid Technologies Conference Europe (ISGT Europe), 2010 IEEE PES, 2010, Page(s): 1- 6.
[7] Z. Liu, W. Gao, Y.-H. Wan, E. Muljadi, "Wind Power Plant Prediction by Using Neural Networks", IEEE Energy Conversion Conference and Exposition. Raleigh, North Carolina September 15-20, 2012

[8] M. A. Kulkarni, S. Patil, G.V. Rama, P.N. Sen, "Wind speed prediction using statistical regression and neural network", J. Earth Syst. Sci. 117, No. 4, 2008, pp. 457-463

[9] Wen-Yeau Chang, Short-Term Wind Power Forecasting Using the Enhanced Particle Swarm Optimization Based Hybrid Method. Energies 2013, 6, 4879-4896; doi:10.3390/en6094879 [10] G. Yang, G. Hu, X. Liu, "A Novel Strategy for Wind Speed Prediction in Wind Farm", TELKOMNIKA, Vol. 11, No. 12, 2013, pp. 7007-7013.

[11] C. Paoli, C. Voyant, M. Muselli, M.L. Nivet, "Forecasting of preprocessed daily solar radiation time series using neural networks", Solar Energy 84, 12 (2010) 2146-2160.

[12] J. Mubiru, E. Banda, "Estimation of monthly average daily global solar irradiation using artificial neural networks", Solar Energy, 82-2, 2008, 181-187.

[13] A. Di Piazza, M. C. Di Piazza, G. Vitale, "Solar Radiation Estimate and Forecasting by Neural Networks-based Approach", XIII Spanish-Portuguese Conference on Electrical Engineering (XIII CHLIE), Valencia, July 3-5, 2013.

[14] A. Di Piazza, M. C. Di Piazza, G. Vitale, "Solar Radiation Estimate and Forecasting by Neural Networks for Smart Grid Energy Management", 28th European PV Energy Conference and Exhibition, Paris, 30 Sept.-04 Oct, 2013.

[15] N. A. Omaima, AL-Allaf, A. Shahlla AbdAlKader, "Nonlinear autoregressive neural networks for estimation soil temperature: a comparison of different optimisation neural network algorithm", UbiCC Jounal, Special Issue of ICIT 2011 conference, 43-51.

[16] Lennart Ljung, System Identification Toolbox ${ }^{\mathrm{TM}} 7$ User's Guide, March 2012. Online only Revised for Version 8.0 (Release 2012a)

[17] H. Holliten, P. Saarikivi, S. Repo, J. Ikaheimo, G. Koreneff, "Prediction errors and balancing costs for wind power production in Finland", Proceedings of the 6th International Workshop on Large-scale integration of Wind Power and Transmission Network for Offshore Wind Farms. Delft, NL, 26-28 Oct.2006.

[18] Mark Hudson Beale, Martin T. Hagan, Howard B. Demuth, Neural Network Toolbox ${ }^{\mathrm{TM}} 7$ User's Guide, September 2010 Online only Revised for Version 7.0 (Release 2010b).

[19] M. Cirrincione, M. Pucci, G. Vitale, "Growing Neural Gas (GNG)-Based Maximum Power Point Tracking for HighPerformance Wind Generator With an Induction Machine", IEEE Trans. On Ind. Appl., vol. 47, no. 2, March/April 2011

[20] M. Y. Uctug, I. Eskandarzadeh, and I. H. Ince, "Modelling and output power optimisation of a wind turbine driven double output induction generator," Proc. Inst. Elect. Eng.-Elect. Power Appl., vol. 141, no. 2, pp. 33-38, Mar. 2004.

[21] E. Koutroulis and K. Kalaitzakis, "Design of a maximum power tracking system for wind-energy-conversion applications," IEEE Trans. Ind. Electron., vol. 53, no. 2, pp. 486-494, Apr. 2006. 